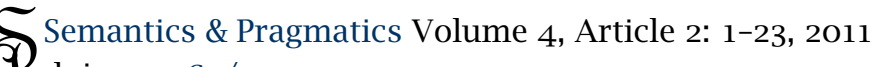

O doi: $10.3765 / \mathrm{sp} .4 .2$

\title{
Exhaustivity in questions with non-factives *
}

\author{
Nathan Klinedinst \\ University College London
}

\author{
Daniel Rothschild \\ All Souls College, Oxford
}

Received 2010-11-29 / First Decision 2011-01-26 / Revision Received 2011-05-16 /

Second Decision 2011-05-25 / Revision Received 2011-06-01 / Published 2011-07-01

\begin{abstract}
This paper is concerned with the conditions under which a person can be said to have told someone or predicted (the answer to a question like) who sang. It is standardly claimed that while (i) the true answer must be completely specified, it is not necessary that (ii) it be specified as being the complete answer. Here the non-factive verbs tell and predict are said to differ from the factive verb know, which typically does impose the strong exhaustivity requirement in (ii). We argue for an intermediate reading of tell and predict that requires more than (i) but less than (ii). To account for this reading we claim that the exhaustivity requirement (ii) imposed by know is due to an operator than can apply non-locally. Applying the operator above a non-factive verb derives the intermediate reading, whereas doing so is vacuous in the case of factives. Thus, we derive the intermediate reading, and differences in the exhaustivity requirements imposed by factives and non-factives, without lexical stipulation.
\end{abstract}

Keywords: questions, exhaustivity, implicature, focus, type-shifting

\section{Introduction}

In this paper we consider the conditions under which a person can be said to have told someone or predicted (the answer to a question like) who sang. It is standard in the literature to distinguish between two possible readings of sentences with embedded questions, weakly exhaustive and strongly exhaustive readings. The weakly exhaustive reading of a question like John predicted

* We are grateful to Emmanuel Chemla, Paul Égré and Benjamin Spector for discussion of aspects of this paper. We are also grateful to an audience at a UCL syntax lunch for discussion, and to two anonymous reviewers at S\&P and the editors-in-chief, for detailed comments.

(C) 2011 Klinedinst \& Rothschild

This is an open-access article distributed under the terms of a Creative Commons NonCommercial License (creativecommons.org/licenses/by-nc/3.o). 
who sang requires that for every person who sang, John predicted that they sang. The strongly exhaustive reading imposes the additional requirement that for everyone who didn't sing John predicted that they didn't sing. It is generally thought that while embedded questions under know only have a strongly exhaustive reading, embedded questions under tell or predict typically get weakly exhaustive readings. We argue here for an intermediate reading of embedded questions under tell and predict that is stronger than the weakly exhaustive reading but weaker than the strongly exhaustive reading.

For example, consider the sentence I predicted who would laugh in a situation where John and Mary laughed and nobody else did. The intermediate reading we propose would require (i) that the speaker predicted that John and Mary would laugh and (ii) that the speaker did not predict that anyone else would laugh. By contrast, the weak reading requires only (i) to hold, while the strongly exhaustive reading also requires that the speaker predicted that nobody other than John and Mary would laugh, a requirement which is stronger than (ii).

We propose to account for both the strongly exhaustive and the intermediate readings by means of a single, independently attested, operator. Applying the operator below the question-embedding verb gives us the strongly exhaustive reading in all cases. Applying the operator above a non-factive verb derives the intermediate reading, whereas doing so has no effect on factive verbs. Thus we derive the intermediate reading (and others attested), while maintaining a uniform semantics for questions, and without positing additional lexical entries for non-factive verbs.

\section{Karttunen and Groenendijk \& Stokhof}

Karttunen (1977) proposed that the semantic denotation of a question like who sang is the set of all true answers to the question. If Frank and Emilio are the only individuals that sang, then the only true answers to the question, on Karttunen's framework, are that Frank sang and that Emilio sang. Departing from the letter, if not the spirit of Karttunen, suppose that when a question is embedded under a proposition-embedding verb like know, the type mismatch is resolved by converting the set of propositions in Karttunen's denotation of the question into the single proposition that is the conjunction of all these propositions. We will call this proposition the K-denotation of the question. Consider, for example, the following: 
Exhaustivity in questions

(1) Arthur knows who sang.

On such a semantics this is true iff Arthur knows for every $x$ who sang that $x$ sang. To make things concrete, we can consider questions such as who sang to be functions from worlds to propositions, i.e., functions of type $\langle s, s t\rangle$. In this case, the semantic value of a question is a function from worlds to the K-denotation:

(2) $\quad \llbracket$ who sang $\rrbracket=\lambda w \cdot \lambda w^{\prime} . \forall x\left(x\right.$ sang in $w \rightarrow x$ sang in $\left.w^{\prime}\right)$

This semantics forms a part of a unified treatment of verbs like know that embed both propositions and questions. Suppose we have a semantics for what we will call know $_{P}$, the proposition-embedding version of know, which takes a proposition, and an individual and returns a new proposition, i.e., $\llbracket$ know $_{P} \rrbracket=\lambda P . \lambda x . \lambda w \cdot x$ knows $P$ in $w$. Then the semantics for $k n o w_{Q}$, the question-embedding know, can be stated in terms of know $w_{P}$ as follows:

(3) $\quad \llbracket \operatorname{know}_{Q} \rrbracket=\lambda Q \cdot \lambda x \cdot \lambda w \cdot \llbracket \operatorname{know}_{P} \rrbracket(Q(w))(x)(w)$

This method can be used uniformly for all question-and-proposition-embedding verbs, such as tell, predict and so on. This theory is both theoretically elegant and makes sharp predictions about the meaning of sentences with embedded questions.

Groenendijk \& Stokhof (1984) criticized this account, inter alia, for failing to capture the strong exhaustivity of embedded questions. They argued that to know who sang one needs not just to know of everyone that sang that they sang but also to know that those are the only people who sang. This claim about truth-conditions is supported by these sorts of cases:

(4) Frank and Emilio are the only students who sang. Arthur knows that Frank and Emilio sang, but he also wrongly believes that Bill and Ted sang. In this case it is wrong to say that Arthur knows who sang. It seems more plausible to say that Arthur doesn't know who sang.

G\&S pointed out that Karttunen's treatment of embedded questions does not handle cases like (4). Using the K-denotation, knowing who sang just requires knowing for every $x$ that did sing that they did sing (this is typically called a weakly exhaustive reading). It does not impose any requirement on the rest of the domain. 
Considerations like the above, motivated G\&S to give their partition semantics for questions. G\&S associated with the question who sang the partitioning of logical space according to the extension of sang. So, any two possible worlds are in the same cell of the partition when the same people sang in both worlds. G\&S proposed that for question-embedding verbs that take propositions, like know, we convert the partition to the proposition expressed by the cell of the partition that the actual world is in. We will call this cell the G\&S-denotation (though this is what G\&S call the extension of a question). So if Frank and Emilio are the only people who sang in the actual world, the G\&S-denotation is the proposition that is true in a world iff Frank and Emilio are the only two people who sang in that world. In the scenario we considered, then, (1) is true iff Arthur knows that Frank and Emilio sang and that no one else did. The G\&S semantics for questions can be expressed as a function (of type $\langle s, s t\rangle$ ) from worlds to G\&S-denotations:

(5) \who sang $\rrbracket=\lambda w \cdot \lambda w^{\prime} \cdot \forall x\left(x\right.$ sang in $w$ iff $x$ sang in $\left.w^{\prime}\right)$

With this semantics we can then use the same rule, (3), to capture the connection between proposition-embedding uses of know and question-embedding uses. This semantics, then, explains the situation in (4).

\subsection{Problems for G\&S with non-factives}

Heim (1994) argued for the utility of the K-denotation by pointing out (i) that the G\&S-denotation could be derived from the K-denotation by a simple operation, ${ }^{1}$ and (ii) that the K-denotation was plausibly needed to capture the truth conditions of some question-embedding verbs. ${ }^{2}$ Evidence for (ii) comes from the particular case of the non-factive verbs tell and predict. ${ }^{3}$ The following cases are to be considered, as above, in a situation in which Frank

1 In particular, if \who $\operatorname{sang}_{K} \rrbracket$ is as in (2) then we can define the G\&S semantics in terms of

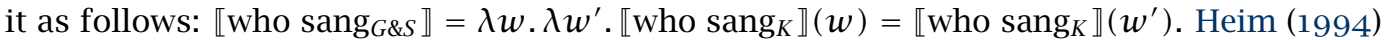
points out there are certain special circumstances where this definition does not exactly match that in (5), but as Sharvit (2002) argues, successfully in our minds, these cases arise because of general problems with possible world semantics rather than anything specific to questions.

2 Heim builds on observations by Berman (1991). Beck \& Rullmann (1999), following Heim, expand the case for (ii).

3 Note that here and below we are focusing on the veridical reading of tell wh-and predict wh-: where what is told or predicted is true. There is also a non-veridical reading, on which, for instance, John predicted who came does not imply that he correctly predicted who came. 
Exhaustivity in questions

and Emilio were the only people who sang:

(6) If John says to me, "Frank and Emilio sang," then he has, in a sense, told me who sang even if he does not say that no one else sang.

(7) If John predicted that Frank and Emilio would sing, then he has, in a sense, predicted who sang even if he made no predictions about whether others would sing.

Examples (6) and (7) suggest that the G\&S-denotation is too strong for tell and predict. Of course, this conclusion would not be warranted if the judgments in (6) and (7) could be shown to ride on an implication or assumption that John's statement/prediction itself was strongly exhaustive. This does not seem to be the case, however, as can be appreciated by fleshing out the scenarios in (6) and (7) as follows. Suppose a game was played, in which John had to guess "sing," "not sing" or "not sure" for each relevant individual. A prize was to be awarded if the singers, but no non-singers, were indicated as "sing." Similarly, a prize was to be awarded if the non-singers, but no singers, were indicated as "not sing." If for example John indicated "sing” for Frank and Emilio and "not sure" for everyone else, it seems correct to say that he gets a prize because he told me/predicted who sang. Similarly, (8) seems true.

(8) John told me/predicted who sang, but did not tell me/predict who did not sing.

Given a fixed domain a telling/prediction of the G\&S-denotation of who sang equates to a telling/prediction of the G\&S-denotation of who did not sing. So it's unclear how (8) could be true, let alone non-contradictory, if only the G\&S-denotation were available. The K-denotation, on the other hand, seems to yield the right results for (6)-(8). ${ }^{4}$

\subsection{Inadequacy of K-denotation for non-factives}

While the considerations in (6) and (7) might seem to argue for the Kdenotation over the G\&S-denotation for the standard readings of non-factives, things are not nearly so simple. There are equally compelling reasons for thinking that simply using the K-denotation with tell and predict will not work. Here are some cases (still to be considered in a scenario where only

4 Karttunen (1977) uses cases like (8) to motivate the K-denotation over a stronger denotation. 
Frank and Emilio sang):

(9) John says to me, "Frank and Emilio sang. And Ted sang as well." In this case, while John has told me the proposition in the K-denotation, it is not at all clear that he has told me who sang.

(10) John predicts that Frank, Emilio and Ted will sing. In this case, John has predicted the K-denotation proposition, but there is a clear sense in which he has failed to predict who sang.

Now, this might be taken as evidence for a simple ambiguity approach: perhaps the intuitions in (9) and (10) arise because the question can be interpreted with the G\&S-denotation, in addition to being interpreted with the K-denotation as judgments like (6) and (7) show. We do think that there are strongly exhaustive readings of tell and predict (see also Heim 1994, Beck \& Rullmann 1999). However, following observations by Spector (2005, 2006), it seems to us that there is an additional, intermediate sense of tell who sang and predict who sang that is at once non-exhaustive in the sense revealed by (6) and (7), and exhaustive in the sense of (9) and (10). This sense only requires the K-denotation, and not the G\&S-denotation, to be told/predicted, but it also requires that no actually false propositions of the form $x$ came were claimed or predicted in error (as in (9) and (10)).

In order to lend some support to these intuitions we conducted a small experiment which we summarize in the appendix: the results suggest that the intermediate reading is spontaneously used by native speakers to determine the truth of embedded questions under predict. (The results also support the existence of weakly and strongly exhaustive readings. $)^{5}$

This view about the truth-conditions of embedded questions is also supported by judgments in cases involving VP ellipsis:

(11) Arthur and Bert write down what they think will be the outcome of tonight's singing talent show, indicating their thoughts on each contestant and what they'll do. Arthur is certain that Frank and Emilio will sing, but not sure whether Bill and Ted will. Bert indicates, perhaps speculating wildly, that each of the four will. It seems true that Arthur predicted who sang, but Bert didn't.

5 The experiment is far from conclusive: it was only meant to lend some support to a judgment we found compelling beforehand. 
Exhaustivity in questions

(12) A phone survey is taken to assess audience interest in last night's episode of a televised talent show, by checking their recall of the contestants. Arthur says, "I'm sure Frank sang and Emilio sang, and I'm not sure about anyone else." Bert says, "Frank, Emilio, Bill and Ted sang." It is decided to send a thank-you prize to Arthur but not Bert on the grounds that it's true that Arthur told us who sang, but Bert didn't.

Positing a K-denotation reading or a G\&S-denotation reading for both the antecedent and elided VP does not account for the true judgments. And while positing a K-reading for the antecedent VP and a G\&S-reading for the elided one would yield the right truth value, there is independent reason to think that this mixed reading is not a possibility. In general, where there is an ambiguity in the antecedent for an elided VP, that ambiguity must be resolved in the same way in both of the VPs. If John went to a bank and Bill did too, either both went to a river or both to a financial institution. ${ }^{6}$ The intermediate sense as described above, however, gets things right: while both Arthur and Bert indicated the K-denotation to be true, only Arthur made no false predictions regarding the question who came.

\section{Intermediate readings}

Thus, what is apparently wanted is a way of generating a reading for tell who sang and predict who sang (etc.) that is intermediate between what the K-denotation for the question gives and what the G\&S-denotation does. An adequate semantics for questions and question-embedding verbs needs to account for these intermediate readings in a principled way.

Most of the literature does not even recognize intermediate readings for predict; Beck \& Rullmann (1999) have room only for weakly exhaustive and strongly exhaustive readings, while Sharvit (2002) seems to think that predict only has a weakly exhaustive reading. For tell, Heim (1994: 137) acknowledges something like the intermediate reading, and suggests that it reflects a unique lexical entry for the verb (and not an additional reading of embedded questions). She suggests that $x$ tells $y Q$ might commonly mean ' $x$ causes $y$ to know the G\&S-denotation of $Q$, by asserting the K-denotation of $Q$.' It is possible that this strategy is generalizable to predict also, though it

6 ... or, alternatively, a bad pun is being made. But it's clear that there's no pun intended in (11) or (12). 
is not obvious how to do this. Regardless, this strategy would require positing an ambiguity in verbs like tell and predict that embed both questions and that-clauses. Not only does one need to posit an ambiguity in the meaning of the verb, but the two readings are not, in fact, that clearly related.

A similar point applies to the more general proposal of Spector (2006, 2005). Spector argues for a systematic three-way ambiguity with embedded questions: weakly exhaustive readings, strongly exhaustive readings and our intermediate readings. He proposes to derive each of the readings from the meaning of the question-embedding verb and the K-denotation, by positing a lexical ambiguity in the verbs. However, on Spector's account the different readings of question-embedding verbs are not clearly related to each other: in particular, the derivation of the strongly exhaustive reading seems to bear no relation to the derivation of the intermediate reading. ${ }^{7}$

An appeal of the accounts of Heim (1994), Beck \& Rullmann (1999) and Sharvit (2002) is that they can account for weak vs. strong exhaustivity of embedded questions in a principled way, using only the standard propositionembedding semantics for the question-embedding verb. As Heim pointed out, the G\&S-denotation can be derived straightforwardly from the K-denotation, and thus weak vs. strong exhaustivity can be explained as a principled ambiguity in the question denotation (or an operation in the lexical semantics of a verb that chooses one of the two types of denotation). We lose much of the appeal and explanatory power if there are still further kinds of exhaustivity that cannot be accounted for in this way. What we propose below is a way of extending the basic strategy of Heim to account for the intermediate form of exhaustivity.

\section{Exhaustivity operators and exhaustive readings}

We propose that the intermediate reading - and all others - are derived by the interaction of the embedded question with an exhaustifying operator EXH. ${ }^{8}$ Intuitively, EXH has a semantics like that of only: it entails the sentence it attaches to, as well as the negations of certain (stronger) alternatives. The latter are ultimately determined by the set of possible or congruent answers

7 In addition, Spector does not draw a distinction between factive and non-factive questionembedding verbs, arguing for intermediate readings in both cases. We return below to the case of factives.

8 We are inspired by the silent exhaustivity operator EXH proposed by Groenendijk \& Stokhof (1984) in order to make answers congruent with the question meaning they assumed. 
Exhaustivity in questions

to the embedded question. We make two basic assumptions: (i) EXH can be applied either to an embedded question directly or above the embedding verb, and (ii) the semantic value of a question is the K-denotation. So, there are (at least) two relevant logical forms for sentences of the form $x V Q$ (e.g., John predicted who came):

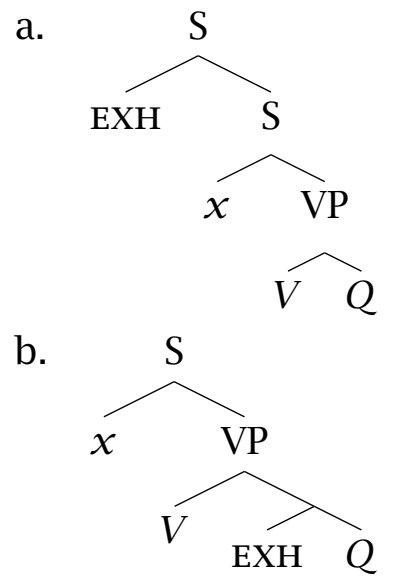

Before discussing the semantics in detail, let us explain informally what these two structures give us. In (13a), EXH applies to the entire sentence: the verb combined with the K-denotation of the question. This structure yields the intermediate readings discussed above. For example, when John predicted who came has the structure in (13a) we get these truth conditions:

(14) John predicted $K$ (= the K-denotation of who came) but did not predict any possible answer $K^{\prime}$ to who came that does not entail $K$.

$\approx$ For every $x$ that came, John predicted that $x$ came, and for no $x$ that did not come did John predict that $x$ came.

This reading is logically distinct from the reading that results when predict embeds either the K-denotation or the G\&S-denotation. On the other hand, the result of applying EXH directly to the embedded question, as in (13b), is simply the G\&S-denotation. Thus, this structure derives the standard reading of sentences with know, which motivated G\&S's proposal. Our proposal can be viewed as a generalization of Heim's (1994) proposal that the K-denotation is basic, and that exhaustive interpretations, where they arise, are derived from the K-denotation via a semantic operation. We depart from Heim in that she only applies a semantic operation directly to the K-denotation, while our EXH can apply non-locally. 
In the remainder of this section we introduce the semantics of EXH, illustrating how it derives the different readings for tell and predict. We then apply the account to factive verbs like know and discuss further predictions. Let us begin with the simple case of the weakly exhaustive reading. We will assume the same semantics for questions and question-embedding verbs we attributed to Karttunen in §2:

(15) $\quad \llbracket$ who came $\rrbracket=\lambda w \cdot \lambda w^{\prime} . \forall x\left(x\right.$ came in $w \rightarrow x$ came in $\left.w^{\prime}\right)$

(16) 【John told me/predicted $Q \rrbracket$ $=\lambda w$. John predicted in $w$ the proposition $\llbracket Q \rrbracket(w)$

Recall that this semantics will get us a weakly exhaustive reading for John told me/predicted who came (assuming there are no hidden operators).

Next consider the strongly exhaustive reading, and the structure in (13b). Readers familiar with Groenendijk \& Stokhof 1984 may be surprised by our proposal that the ExH operator should be able to apply directly to a question as in (13b). G\&S introduced this operator to apply to answers rather than questions; for questions G\&S treat the strongly exhaustive semantics (the G\&S-denotation) as basic. Their exhaustivity operator was introduced to model the fact that answers are (typically) interpreted as complete or exhaustive. ${ }^{9}$ We depart from them here: we derive strongly exhaustive readings for questions from the K-denotation by means of their EXH operator, suitably generalized. In order to do this, we need to do two things: (i) specify the focus set for questions, and (ii) show how a propositional operator like EXH can apply to questions which are of a higher type.

We turn to (i) first. A question like who came we associate not only with a denotation, but with a focus value as well (Rooth 1985). Since the normal semantic value of who came is a function from worlds to propositions (the K-denotation), we will take its focus value, $\llbracket$ who came $\rrbracket_{F}$, to be a set of objects

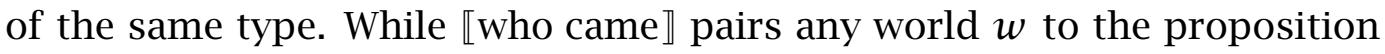
that is the (non-exhaustively) true answer to who came in $w$, an element of the focus value of who came pairs $w$ to some possible or congruent answer. Formally, this can be expressed as follows:

(17) $\llbracket$ who came $\rrbracket_{F}=\left\{Q^{\prime}: \forall w \exists w^{\prime}\left(Q^{\prime}(w)=\llbracket\right.\right.$ who came $\left.\left.\rrbracket\left(w^{\prime}\right)\right)\right\}$

9 Hence, the title of their dissertation Studies in the Semantics of Questions and the Pragmatics of Answers (our emphasis). Thus they built strongly exhaustive readings into the semantics of questions, but for answers they derive a strongly exhaustive interpretation by the silent (semi-)pragmatic operator EXH. 
Exhaustivity in questions

We call $\llbracket$ who came $\rrbracket_{F}$ a focus value because of its similarities to standard focus values in the literature; for example, the members of $\llbracket$ who came $\rrbracket_{F}$ applied to any world $w$ are just the possible or congruent answers to who came. However, it is not clear to us how well this will integrate with the general theory of focus. In this respect we follow the scalar implicature literature (e.g., Sauerland 2004, Spector 2006), where focus-like structures (i.e., sets of alternatives) are used but not necessarily identified with standard focus values.

We now turn to (ii), defining the ExH operator in such a way that it can apply to both propositions, type $\langle s t\rangle$, and questions, type $\langle s, s t\rangle$. This requires first a general definition of the logical connectives and operators $\supseteq$, $\wedge$, and $\neg$ that will apply to functions from worlds to propositions. We use the standard generalizations from the type-shifting literature (Partee \& Rooth 1983, Groenendijk \& Stokhof 1988): ${ }^{10}$

$$
\begin{gathered}
P \supseteq Q=\operatorname{def}\left\{\begin{array}{l}
Q \rightarrow P, \text { if } P \text { and } Q \text { are of type } t \\
\forall x[P(x) \supseteq Q(x)], \text { otherwise }
\end{array}\right. \\
P \wedge Q=\operatorname{def}\left\{\begin{array}{l}
P \& Q, \text { if } P \text { and } Q \text { are of type } t \\
\lambda x \cdot P(x) \wedge Q(x), \text { otherwise }
\end{array}\right. \\
\neg P=\operatorname{def}\left\{\begin{array}{l}
\sim P, \text { if } P \text { is of type } t \\
\lambda x \cdot \neg P(x), \text { otherwise }
\end{array}\right.
\end{gathered}
$$

These generalized connectives are defined in the most straightforward way in terms of the propositional ones. For example, where $\alpha$ and $\beta$ are functions from worlds to propositions, their generalized conjunction, $\alpha \wedge \beta$, is simply the function that pairs any world $w$ to the propositional conjunction of $\alpha(w)$ and $\beta(w)$. And the generalized negation of $\alpha, \neg \alpha$, is just the function that pairs any world $w$ to the propositional negation of $\alpha(w)$.

As noted above, EXH has a semantics like that of only: it entails (in the generalized sense) the sentence $\phi$ that it is prefixed to, as well as the (generalized) negations of certain stronger alternatives to $\phi$. The latter we take to be the members of a subset, call it $\llbracket \phi \rrbracket_{F^{+}}$, of the focus value of $\phi$, so that EXH is defined as follows: ${ }^{11}$

$$
\llbracket \mathrm{EXH} \phi \rrbracket=\llbracket \phi \rrbracket \wedge\left(\bigwedge_{P \in \llbracket \phi \rrbracket_{F^{+}}} \neg P\right)
$$

10 We use $\rightarrow, \&$, and $\sim$ to represent the standard truth-functional connectives.

11 Where $S$ is a set $\left\{\phi_{1}, \ldots \phi_{n}\right\}$ of functions of appropriate type, we write $\Lambda_{\phi \in S} \phi$ for the generalized conjunction $\phi_{1} \wedge \cdots \wedge \phi_{n}$ of its members. 
We return below to discussing the general definition of $\llbracket \rrbracket_{F^{+}}$. For the particular case of a question like who came, we assume the following:

$$
\llbracket \text { who came } \rrbracket_{F^{+}}=\left\{Q^{\prime} \in \llbracket \text { who came } \rrbracket_{F}: Q^{\prime} \supset \llbracket \text { who came } \rrbracket\right\}
$$

So $\llbracket$ who came $\rrbracket_{F^{+}}$is just the subset of focus alternatives to who came that are strictly stronger, in the generalized sense, than the K-denotation. In other words, $\llbracket$ who came $\rrbracket_{F^{+}}$contains those elements of $\llbracket$ who came $\rrbracket_{F}$ that pair each $w$ to a possible answer to who came which is stronger than the K-denotation in $w$.

Consider now EXH applied directly to the question who came. By the

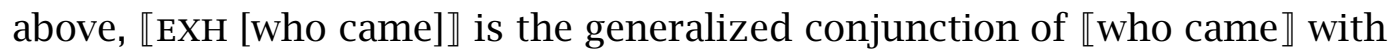
each of the generalized negations of its stronger alternatives:

(23) $\llbracket$ who came $\rrbracket \wedge\left(\bigwedge_{P \in \llbracket \text { who came }]_{F^{+}}} \neg P\right)$

By the above definitions, (23) amounts to the following:

(24) $\lambda w \cdot \lambda w^{\prime}$.

$\forall x\left(x\right.$ came in $w \rightarrow x$ came in $\left.w^{\prime}\right) \wedge\left(\bigwedge_{P \in[\text { who came }]_{F^{+}}} \neg P(w)\left(w^{\prime}\right)\right)$

Example (24) is the function that maps any $w$ to the proposition that is true in a world $w^{\prime}$ iff (i) the K-denotation in $w$ holds in $w^{\prime}$, but (ii) no possible answer to who came holds in $w^{\prime}$ that is stronger than the K-denotation in $w$. That is, (24) is a function that pairs any $w$ to the set of worlds that agree with $w$ on the the extension of came. The latter is just the G\&S-denotation of who came in $w$, and so we derive a strongly exhaustive reading from applying EXH locally to the embedded question (by (16)), as in this example:

(25) John told me/predicted [EXH [who came]]

We now turn to intermediate readings. To derive these we will apply EXH above the level of the question, and so we need an appropriate focus value, and set of stronger focus alternatives, for John told me/predicted who came. We will not give a formal recursive definition of focus values here; rather we assume the standard definition from Rooth 1992, 1997. The intuitive idea is that the focus values of wholes are determined from those of their parts by point-wise combining the focus values of the parts. Where an expression is neither conventionally/lexically, nor intonationally marked for focus, its focus value is simply its normal denotation. Given that the focus value of who came is as in (17), these principles yield the following, which we will 
Exhaustivity in questions

simply assume:

【John predicted $Q \rrbracket_{F}$ $=\left\{\lambda w\right.$. John predicted in $w$ the proposition $\left.Q^{\prime}(w): Q^{\prime} \in \llbracket Q \rrbracket_{F}\right\}$

That is, the focus value of John told me/predicted who came is the set of propositions that attribute to John the telling/prediction of some pattern of possible answers to who came. The relevant set of stronger focus alternatives, 匹 John predicted $Q \rrbracket_{F^{+}}$, we take to be as follows:

(27) $\llbracket J o h n$ predicted $Q \rrbracket_{F^{+}}$ $=\left\{\lambda w\right.$. John predicted in $w$ the proposition $\left.Q^{\prime}(w): Q^{\prime} \in \llbracket Q \rrbracket_{F^{+}}\right\}$

This amounts to the set of predicting profiles for John where in each world he predicts a strictly stronger possible answer to $Q$ than the one that is actual in that world. (27) could be derived by adopting a compositional definition of $\llbracket \rrbracket_{F^{+}}$that simply mirrors Rooth's definition of $\llbracket \rrbracket_{F}$, i.e., based on pointwise combination of lexically specified values of $\llbracket \rrbracket_{F^{+}}$. However, we believe that a more principled definition in terms of $\llbracket \rrbracket_{F}$ itself can also be given. ${ }^{12}$

Now we derive the intermediately exhaustive reading by applying EXH to (28), as in (29).

(28) John predicted who came.

(29) EXH [John predicted who came]

By the definition of $\mathrm{EXH}$, in (21), we derive the following:

(30) $\llbracket$ EXH [John predicted who came $] \rrbracket$

$$
=\llbracket \mathrm{John} \text { predicted who came } \rrbracket \wedge\left(\bigwedge_{P \in \llbracket \text { John predicted who came } \rrbracket_{F^{+}}} \neg P\right)
$$

12 The rule needed is as follows:

$$
\llbracket \alpha \rrbracket_{F^{+}}=\left\{x \in \llbracket \alpha \rrbracket_{F}: \forall x^{\prime} \in \llbracket \alpha \rrbracket_{F}\left(\llbracket \alpha \rrbracket \supseteq x^{\prime} \vee \neg\left((\llbracket \alpha \rrbracket \wedge x) \supseteq x^{\prime}\right)\right)\right\}
$$

This definition makes use of the idea of innocent excludability from Sauerland (2004) and Fox (2007). The basic idea is that the relevant set of stronger alternatives $F^{+}$is the set of all those alternatives which can be negated without automatically including some other stronger alternative. In the case of $\llbracket$ who came $\rrbracket_{F}$ using this definition $\llbracket$ who came $\rrbracket_{F^{+}}$turns out to be the set of all functions that map any world $w$ to a proposition that is strictly stronger than $\llbracket$ who came $\rrbracket(w)$, which is what we have in our (22). For $\llbracket$ John predicted $Q \rrbracket_{F}$ the only innocently excludable alternatives are those propositions which are true in a world $w$ only if John predicted a proposition strictly stronger than $\llbracket Q \rrbracket(w)$. 
Here $\wedge$ and $\neg$ apply directly to propositions (i.e., things of type $\langle s t\rangle$ ) and so have their customary interpretation). (30) is thus the proposition that is true in a world $w$ iff (i) $w$ is in the denotation of the John predicted/told me who came, and (ii) $w$ is not in any of the stronger alternatives to this. Condition (i) is satisfied iff John predicted in $w$ the K-denotation in $w$, as we argued earlier. Condition (ii) is satisfied just in case John did not predict any stronger possible answer to who came than the K-denotation in $w$. The two conditions combined then amount to our intermediate reading: (30) is the proposition that John predicted the K-denotation, but did not predict any stronger possible answer.

It is apparent in the simple case just illustrated that the intermediate reading is something like a K-denotation reading strengthened by a scalar implicature computed from a scale of alternative answers. For, what EXH effectively does in (29) is rule out the possibility that John told/predicted any possible answer to who came that is logically stronger than the K-denotation. If this is right, deriving the intermediate reading is a bit like inferring from the claim that John did some of the work that John did not do all of the work, i.e., it is like a normal scalar implicature. This might suggest that no modification is actually required to an account like Heim's which includes the K-reading semantically: the intermediate reading is just derived by ordinary, Gricean scalar implicature from the K-reading.

However, we believe that the intermediate reading cannot be explained away as a scalar implicature in a way that threatens our proposal. One reason for thinking this is that the intermediate reading persists in downwardentailing environments while standard scalar implicatures do not. This point is already illustrated by (11) and (12), where getting the correct truth judgment by implicature would require computing the putative scalar implicature in the scope of the negation in the second conjunct. The following pair illustrates the distinction here between intermediate readings of questions and normal scalar implicatures in downward-entailing environments:

(31) If you predict who sang, you win a hundred dollars. can mean: If you ExH predict who sang, you win a hundred dollars. (i.e., predict can have the intermediate reading)

(32) If you do some of the work, you get fired. cannot mean: If you do some but not all the work, you get fired. 
Of course, it has been argued from similar considerations that certain phenomena related to "scalar implicatures" are, in fact, not Gricean/pragmatic but rather due to the grammatical presence of exhaustivity operators like the one we posit (e.g., Chierchia, Fox \& Spector forthcoming). The critical claim of our paper is that we can derive both the strongly exhaustive reading and the intermediate reading using exhaustivity operators and the K-denotation for questions (with its focus values as above). What would seem strange to us is to treat the existence of the G\&S-reading as the result of a semantic ambiguity while treating the existence of the intermediate reading as the result of a pragmatic process. By contrast it is entirely consonant with our proposal to derive both these readings with the same exhaustivity operator responsible for scalar implicatures more generally. ${ }^{13}$

We now turn to factive question-embedding verbs such as know, where the effects of EXH are somewhat different. Consider, first, the wide-scope application of the EXH operator:

(33) John knows who came.

(34) EXH [John knows who came]

By the definition of EXH in (21), we derive the following:

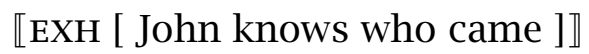

$$
=\llbracket \text { John knows who came } \rrbracket \wedge\left(\bigwedge_{P \in \llbracket \text { John knows who came } \rrbracket_{F^{+}}} \neg P\right)
$$

It turns out that (33) is identical to (34), so the wide-scope EXH has no effect. To see this note that for a world $w$ to be in (35) it needs to satisfy two conditions: (i) John knows the K-denotation in $w$, and (ii) there is no possible answer stronger than the K-denotation such that John knows it. What we need to argue is that (ii) rules out no more worlds than (i) alone does. Suppose John knows the K-denotation in a world $w$. Then John knows for everyone who came in $w$ that they came. Now consider any stronger possible answer $P$ in $w: P$ will be a proposition that not only entails the K-denotation but also

13 Obviously if the exhaustivity of questions is to be part of a more general grammatical theory of scalar implicature, we will need to explain why question exhaustivity persists in downward-entailing environments while normal scalar implicatures do not. One possible explanation is the inherent focus values of questions. It is often observed that normal scalar implicatures are more easily found in downward-entailing environments when focus is used. For example, the relevant reading of (32) might be possible if some is focused. Benjamin Spector (personal communication) suggested to us that a similar explanation might account for the persistence of exact readings of numerals in downward-entailing environments. 
entails that at least one other $x$ person came. We can immediately note that $P$ is false in $w$ : the K-denotation, by definition, is the strongest true answer, so anything stronger is false. But since $P$ is false in $w$, it follows from the factivity of knowledge that John does not know $P$ in $w .^{14}$ So, condition (ii) is automatically satisfied whenever condition (i) is. It follows that (35) and (33) are equivalent.

On the other hand, if we allow EXH to apply directly to the question as in (36), we get a sentence that is true iff John knows the G\&S-denotation, the standard reading reported in the literature.

(36) John knows EXH [who came]

It seems plausible to us that this is the only exhaustive reading for know.

\section{Summary and issues}

We argued for a third kind of exhaustivity, intermediate exhaustivity, not captured by current accounts. We proposed to account for this reading and the other two standard ones using just Karttunen's denotation for questions and an exhaustivity operator. Our method for doing so, moreover, does not require positing additional lexical entries for question-embedding verbs as suggested, for instance, by Heim for tell. On our view, those verbs that embed both questions and that-clauses make the same semantic contribution in all cases. We conclude with a brief discussion of some refinements to our basic account that are necessary to deal with more complex examples.

Scope of EXH We presented EXH as an operator that could apply to a constituent of any type ending in $t$. However, as far as we can tell, it does not combine freely with any expression. Consider the following:

(37) At least one student predicted who came.

Applying EXH to the top of (37) gives a proposition whose truth requires, among other things, that no student made any actually false prediction about who came. It seems unlikely that such a reading is available. Thus the scope of EXH in a main clause seems to be limited to the VP level, beneath any

14 We assume here, for simplicity, a bivalent semantics for know: $x$ knows $P$ is false if $P$ is false. Despite the factive presuppositions of know, many argue that it has a bivalent meaning (e.g., Stalnaker 1974). 
Exhaustivity in questions

quantifiers or operators. There are various ways to enforce this requirement but we will not make a choice here. ${ }^{15}$

Factivity As we mentioned above, Spector proposes intermediate readings for factive question-embedding verbs. For example, in his discussion of intermediate readings Spector (2006: ch. 2) uses French deviner (meaning roughly foretell), which appears to be factive unlike predict. Thus, he claims that factive verbs can have intermediate readings, something our account does not naturally allow. His basic claim (stated for English foretell rather than French deviner) is that (38a) has the truth-conditions in (38b).

(38) a. It was foretold who would come.

b. For every $x$, if $x$ came, it was foretold that $x$ came, and if $x$ didn't come, it wasn't predicted that $x$ would come.

If (i) foretell and deviner are factive and (ii) this intermediate reading exists then this is something our theory does not account for. However, note that if (i) and (ii) are really true then the intermediate readings for these verbs are not paraphrasable without the use of a separate verb besides foretell and deviner. For example, the second clause of $(38 \mathrm{~b})$ needs to use predict rather than foretell if we assume foretellings cannot be false, otherwise it is trivial. This suggests some form of lexical decomposition, which would open up a number of avenues for treating these cases within the framework we give here. ${ }^{16}$

De dicto readings Groenendijk \& Stokhof (1984) claimed another major empirical advantage over Karttunen's approach: their semantics captured what they call the de dicto readings of embedded questions. For these it is best to think of questions where the wh-phrase contains a predicate, for example which-questions such as the following:

15 For example, it could be assumed that in main clauses EXH must combine with the most embedded proposition-denoting expression, a VP containing a subject trace (as suggested in the tree $(13 a))$.

16 We are grateful to Paul Égré and Benjamin Spector for bringing this issue to our attention. See George 2010 for a critique of Spector's proposed lexical entry for intermediate readings for factives, and discussion of lexical decomposition. It's worth nothing that Berman (1991) and Spector (2006) also suggest that there are intermediate readings for know (e.g., to know who came $=$ for all $x$, if $x$ came to know $x$ came, and if $x$ didn't come to not believe $x$ came). The same point about lexical decomposition applies here. 
(39) Which students sang?

Suppose Frank and Emilio were the only students who sang. Knowing either the K-denotation or the G\&S-denotation of (39) does not require knowing whether any individual is a student or not. G\&S and others have argued there are cases where knowing which students sang requires some knowledge about who is a student. Here is the sort of scenario that motivates this view:

(40) John knows who sang at the concert last night. However he has no idea who was a student, so he doesn't know which students sang.

G\&S propose to account for this reading by giving a distinct, de dicto partition for questions and proposing that embedded questions are semantically ambiguous. Their de dicto reading of questions of the form which Fs are $G$ partitions the space of possible worlds by the extension of $F \wedge G$. So, to know which $F$ s are $G$ in the de dicto sense is to know for every $x$ in the intersection of $F$ and $G$ that it is in the intersection of $F$ and $G$, and to know that no other individual satisfies both $F$ and $G$. The proposition one knows in this case is the G\&S de dicto denotation of the question.

This de dicto denotation of questions can be easily captured in the framework we used in the last section by positing an ambiguity in the semantic value and the focus value of questions. Our de re semantics for questions generalizes as follows to questions of the form which Fs are G:

(41) 【which $F_{\text {de } r e}$ is $G \rrbracket$

$$
=\lambda w \cdot \lambda w^{\prime} . \forall x\left[F x \text { in } w \rightarrow\left(G x \text { in } w^{\prime} \rightarrow G x \text { in } w^{\prime}\right)\right]
$$

Now the variation that gives you the de dicto reading goes as follows:

(42) «which $F_{\text {de dicto }}$ is $G \rrbracket$

$$
=\lambda w \cdot \lambda w^{\prime} . \forall x\left(F x \& G x \text { in } w^{\prime} \rightarrow F x \& G x \text { in } w^{\prime}\right)
$$

Using this de dicto semantics for which $F$ is $G$ we get the result that a sentence like (43) will have exactly G\&S's de dicto reading.

(43) John knows EXH [which students sang]

This treatment of the de re/de dicto distinction does not require positing any unexplained semantic ambiguity: we can derive (41) from (42) simply by actualizing the predicate $F$ (i.e., evaluating it relative to a world variable that picks out the actual world). Thus, we can treat the de dicto value as basic and 
Exhaustivity in questions

derive the de re value. ${ }^{17,18}$

\section{Appendix: Survey}

In order to provide evidence for the existence of the intermediately exhaustive reading of questions we conducted a small survey. We gave the survey to 193 subjects (recruited through Amazon's Mechanical Turk), but once we eliminated non-native speakers and those who showed that they did not understand the task by incorrectly answering basic comprehension questions we were left with results from 65 individuals. ${ }^{20}$

We presented subjects with a scenario in which various people had written down predictions about which of four individuals would make the Olympic swimming team. Schematically, there were four possible individuals $a, b, c$ and $d$ trying out for the swimming team. We stipulated that $a$ and $d$ made

17 This is the strategy in Heim 1994: the de re reading is derived from the de dicto reading by actualizing one of the question predictates.

18 Note that we do not link the derivation of de dicto readings to strong exhaustivity in any way: rather we posit a more basic semantic ambiguity in the interpretation of the question predicate $F$. Sharvit (2002) argues against separating de dicto readings from the derivation of the strong exhaustivity, a separation Beck \& Rullmann (1999) make but Heim (1994) does not. On Heim's account, the only way to generate a de dicto reading is to apply her equivalent of the EXH operator. Sharvit (2002) claims that Heim's account is superior since there is a class of verbs, including surprise and predict that (a) only exhibit weakly exhaustive readings (i.e., those given by the K-denotation) and (b) do not allow de dicto readings. ${ }^{19}$ That property (a) would entail property (b) is predicted on Heim's account but would require stipulations on Beck \& Rullmann's (and ours). We think (a) is false: predict and surprise have strongly exhaustive readings.

Here is a an example where surprise shows a strongly exhaustive reading:

(i)

Four students run a race: Bob, Ted, Alice and Sue. Emily expects Bob, Ted and Alice to run it in under six minutes. Only Bob runs it in under six minutes. Emily is surprised who ran the race in under six minutes (since she expected more people to).

However, if surprise only can embed the K-denotation we should not be able to say what we do in (i). This is because if one just embeds the K-denotation in the scenario there then Emily is surprised who ran under six minutes should be true iff Emily is surprised that Bob ran under six minutes. Similar comments can be made about predict. If we are right, then Sharvit's argument is not successful since she provides no evidence that absence of de dicto readings are linked to verbs that only exhibit weakly exhaustive readings, since such verbs do not seem to exist.

19 Claim (a) is on page 112 , claim (b) is on page 116-121.

20 The survey and the results may be found at http://users.ox.ac.uk/ sfopozoo/ questionsurvey/. 


\begin{tabular}{ccccc} 
& $\mathrm{a}$ & $\mathrm{b}$ & $\mathrm{c}$ & $\mathrm{d}$ \\
\hline 1 & No & $?$ & No & Yes \\
2 & $?$ & $?$ & No & Yes \\
3 & Yes & $?$ & No & Yes \\
4 & Yes & Yes & $?$ & Yes
\end{tabular}

Table 1 Predictions

the swimming team and $b$ and $c$ failed to. A prediction consisted of a list of the four names with a "Yes," "No" or "?" next to each name. We asked our subjects to report for four sets of predictions, listed in Table 1, whether or not the set of predictions "correctly predicted who made the swimming team."

Subjects needed to code predictions 1 and 2 both as not correctly predicting who made the swimming team in order to count as using one of the three strategies. 51 of 65 subjects $(78.4 \%)$ did this. The predictions in 3 and 4 together distinguished between the three types of exhaustivity: If the subjects applied a strongly exhaustive interpretation to predict-wh then neither prediction 3 nor prediction 4 would qualify as correctly predicting who made the team. With a weakly exhaustive reading both prediction 3 and 4 would qualify. And with an intermediately exhaustive interpretation 3 would qualify, but 4 would not (since it contains a false prediction). Coding 4 but not 3 as correctly predicting who made the team would not be consistent with any of the senses of exhaustivity discussed. Of the 51 subjects who coded predictions 1 and 2 consistently with one of the three exhaustive readings, 14 answers (27.5\%) were strongly exhaustive, 19 (37.2\%) were weakly exhaustive, 17 (33.3\%) were intermediately exhaustive and only 1 subject (1.9\%) gave the answer inconsistent with any of the types of exhaustivity. That the intermediate reading was attested by so many respondents provides evidence that subjects used this reading rather than just confining themselves to weak and strong exhaustivity. ${ }^{21}$

$21 \mathrm{~A} \chi^{2}$-test shows that this pattern of answers differs significantly from a pattern in which the answers not consistent with weak or strong exhaustivity would be equally distributed (as we would expect if they were generated by noise). The actual pattern of 17, 19, 14, 1 differs significantly from $9,19,14,9,\left(\chi^{2}(3)=14.2, p<.005\right)$. 
Exhaustivity in questions

Of course, given the details of our small study there will be other explanations for the presence of the intermediate reading, but we think these results might provide some support for the judgments we report in this paper as well as suggesting new avenues for the empirical study of question meaning.

\section{References}

Beck, Sigrid \& Hotze Rullmann. 1999. A flexible approach to exhaustivity in questions. Natural Language Semantics 7(3). 249-298. doi:10.1023/A:1008373224343.

Berman, Stephen. 1991. On the semantics and logical form of wh-clauses. Amherst: University of Massachusetts dissertation. http://scholarworks. umass.edu/dissertations/AAI9120852.

Chierchia, Gennaro, Danny Fox \& Benjamin Spector. forthcoming. The grammatical view of scalar implicatures and the relationship between semantics and pragmatics. In Paul Portner, Claudia Maienborn \& Klaus von Heusinger (eds.), Semantics: An international handbook of natural language meaning (Handbooks of Linguistics and Communication Science 33), Berlin: De Gruyter Mouton. http://www.semanticsarchive.net/ Archive/WMzY2ZmY/CFS_EmbeddedSIs.pdf.

Fox, Danny. 2007. Free choice and the theory of scalar implicatures. In Uli Sauerland \& Penka Stateva (eds.), Presupposition and implicature in compositional semantics (Palgrave Studies in Pragmatics, Language and Cognition), 71-120. Houndmills, Basingstoke \& Hampshire: Palgrave Macmillan.

George, Benjamin R. 2010. Responsive predicates and (non-)reducibility. Unpublished manuscript, UCLA. http://brg42.bol.ucla.edu/brg_reducibility. pdf.

Groenendijk, Jeroen \& Martin Stokhof. 1984. Studies in the semantics of questions and the pragmatics of answers. Amsterdam: University of Amsterdam dissertation. http://dare.uva.nl/en/record/123669.

Groenendijk, Jeroen \& Martin Stokhof. 1988. Type-shifting rules and the semantics of interrogatives. In Gennaro Chierchia, Barbara H. Partee \& Raymond Turner (eds.), Properties, types, and meaning, Volume II: Semantic issues (Studies in Linguistics and Philosophy 39), 21-68. Berlin \& Heidelberg: Springer. http://www.springer.com/education+\%26+language/ linguistics/book/978-1-55608-o88-3.

Heim, Irene. 1994. Interrogative semantics and Karttunen's semantics for know. In Rhonna Buchalla \& Anita Mittwoch (eds.), The Israel Associ- 
ation for Theoretical Lingistics (IATL 1), 128-144. Hebrew University of Jerusalem. http://semanticsarchive.net/Archive/jUzYjkıO/Interrogative\% 2094.pdf.

Karttunen, Lauri. 1977. Syntax and semantics of questions. Linguistics and Philosophy 1(1). 3-44. doi:10.1007/BFoo351935.

Partee, Barbara H. \& Mats Rooth. 1983. Generalized conjunction and type ambiguity. In Rainer Bäuerle, Christoph Schwarze \& Arnim von Stechow (eds.), Meaning, use, and interpretation of language (Foundations of Communication), 361-383. Berlin: de Gruyter. http://semanticsarchive.net/ Archive/ThiYWY5Y/BHP_Rooth83Generalized\%20Conjunction.pdf.

Rooth, Mats. 1985. Association with focus. Amherst: University of Massachusetts dissertation. http://scholarworks.umass.edu/dissertations/ AAI8509599.

Rooth, Mats. 1992. A theory of focus interpretation. Natural Language Semantics 1(1). 75-116. doi:10.1007/BFo2342617.

Rooth, Mats. 1997. Focus. In Shalom Lappin (ed.), The handbook of contemporary semantic theory (Blackwell Handbooks in Linguistics 3), 271-297. London: Wiley-Blackwell. ftp://ftp.ims.uni-stuttgart.de/pub/papers/mats/ survey.ps.gz.

Sauerland, Uli. 2004. Scalar implicatures in complex sentences. Linguistics and Philosophy 27(3). 367-391. doi:10.1023/B:LING.00ooo23378.71748.db.

Sharvit, Yael. 2002. Embedded questions and 'de dicto' readings. Natural Language Semantics 10(2). 97-123. doi:10.1023/A:1016573907314.

Spector, Benjamin. 2005. Exhaustive interpretations: What to say and what not to say. Unpublished paper presented at the LSA workshop on Context and Content.

Spector, Benjamin. 2006. Aspects de la pragmatique des opérateurs logiques. Paris: Université Paris Diderot dissertation. http://lumiere.ens.fr/ bspector/THESE_SPECTOR/THESE_SPECTOR_AVEC_ANNEXE2.pdf.

Stalnaker, Robert. 1974. Pragmatic presuppositions. In Milton Munitz \& Peter Unger (eds.), Semantics and philosophy, 197-213. New York: New York University Press. 
Exhaustivity in questions

Nathan Klinedinst

UCL Psychology and Language Sciences

2 Wakefield Street

London

$\mathrm{WC} 1 \mathrm{~N} 1 \mathrm{PF}$

UK

nathank@ucl.ac.uk
Daniel Rothschild

All Souls College

Oxford

OX14AL

UK

daniel.rothschild@philosophy.ox.ac.uk 